Cochrane produit des revues systématiques dédiées à diverses thématiques de la recherche médicale, créant ainsi une base fiable pour la médecine fondée sur les données probantes. Depuis 2016, tous les résidents suisses bénéficient d'un accès libre et gratuit à la Cochrane Library. Dans l'interview de cette semaine, venant clore notre série de quatre articles sur la question, le Dr méd. Erik von Elm, directeur de Cochrane Suisse, se penche sur les chances et les défis que représente l'utilisation de la Cochrane Library.

Dr méd. Christoph Bosshard, vice-président de la FMH, responsable du département Données, démographie et qualité

\title{
Gros plan sur la Cochrane Library
}

\author{
Interview: Dre sc. Stefanie Hostettler
}

\begin{abstract}
Docteur von Elm, vous êtes directeur de Cochrane Suisse. Quelle est la place de Cochrane dans la recherche et qu'est-ce qui vous a incité à collaborer avec Cochrane?

Cochrane s'investit depuis plus de 25 ans pour des revues systématiques permettant d'évaluer et de résumer les résultats de la recherche. Notre travail sert à amener les preuves là où on en a le plus besoin: lors des décisions de santé, et à tous les niveaux. C'est ce qui me motive à contribuer au travail de ce réseau, tout comme les quelques milliers d'autres membres Cochrane dans le monde.
\end{abstract}

\section{Qu'est-ce qui a changé depuis l'introduction de la Cochrane Library?}

Dès le départ, le travail s'est concentré sur le développement et l'amélioration de l'ensemble des revues systématiques de la Cochrane Library. Depuis le début des années 90, Cochrane est passé d'un réseau isolé d'universitaires partageant le même état d'esprit à une organisation internationale dans laquelle interviennent

\section{Cochrane s'investit depuis plus de 25 ans pour} des revues systématiques permettant d'évaluer et de résumer les résultats de la recherche.

non seulement des chercheurs, mais aussi des personnes d'autres horizons, des patients par exemple. La Cochrane Library a contribué à renforcer l'influence de la preuve scientifique sur l'activité médicale.

\section{A qui s'adresse la Cochrane Library?} Comment atteignez-vous les groupes cibles?

Les professionnels de la santé constituent le principal groupe cible. Mais il existe désormais de nombreux résumés dits "en langage clair», non seulement en anglais, mais aussi en français, en allemand et dans

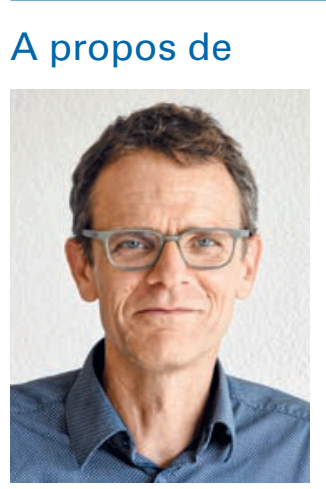

Dr méd. Erik von Elm

MSc, spécialiste en prévention et santé publique

Erik von Elm est le cofondateur et directeur de Cochrane Suisse. Après des études de médecine et un doctorat en Allemagne et en France, il a obtenu un master en épidémiologie à la London School of Hygiene and Tropical Medicine et le titre de spécialiste en prévention et santé publique en Suisse.

II est co-instigateur et auteur d'initiatives visant à améliorer la qualité des articles scientifiques, à l'instar de la déclaration STROBE pour l'élaboration des rapports d'études observationnelles, et expert pour revues médicales. En tant que chef de clinique scientifique, il intervient dans la recherche et l'enseignement au Centre universitaire de médecine générale et santé publique (Unisanté) à Lausanne et enseigne, entre autres, les thèmes relevant des méthodes et de la recherche en médecine clinique et santé publique. Membre élu du Cochrane Council, il y représente les centres nationaux.

de nombreuses autres langues. Les données probantes deviennent ainsi accessibles aux personnes qui ont peu de connaissances médicales. La discussion entre le médecin et son patient peut avoir lieu sans que l'information ait été diffusée de manière unilatérale.

\section{En 2016, l'ASSM, l'OFSP et les bibliothèques universitaires ont financé une licence nationale. \\ Quel a été l'impact?}

L'impact a été important et il suscite l'envie de collègues du réseau Cochrane dans plusieurs pays. Le nombre d'accès a doublé dès le premier jour; il s'est maintenu à un niveau élevé et constant. Nous espérons que cette offre sera toujours plus sollicitée à l'avenir, 
notamment par les personnes qui se posent des questions sur la santé.

Pour les médecins et les professionnels de la santé, quels sont les avantages d'utiliser la Cochrane Library?

Le plus gros avantage est sans aucun doute le gain de temps; plus besoin d'éplucher tous les articles spécialisés, parfois contradictoires, puisque leurs conclusions sont présentées dans un travail recensant l'ensemble des résultats. Pour cela, le format Clinical Answers est très utile et offre un accès rapide aux données probantes.

Le plus gros avantage est sans aucun doute le gain de temps; les conclusions sont présentées dans un travail recensant l'ensemble des résultats.

Le Dr Bruno Kissling, médecin de famille, montre dans son article que les questions relevant spécifiquement de la médecine générale sont souvent complexes, car plusieurs problèmes médicaux se superposent. Que peut faire la Cochrane Library pour assister les médecins dans ce genre de situations très spécifiques?

L'analyse de la situation d'un patient ne s'improvise pas; il faut l'expérience du spécialiste pour ordonner les problèmes de santé et les pondérer. Jusqu'à présent, aucune source d'information médicale n'a traité de manière satisfaisante la complexité liée par exemple à la polymorbidité, ni les nouvelles technologies comme l'intelligence artificielle, dont l'utilisation en est encore à ses débuts. En revanche, si on a un ensemble de questions concrètes, on peut consulter la Cochrane Library et vérifier s'il existe suffisamment de preuves sur l'efficacité d'une médication, par exemple. Un défaut de preuves pourra même permettre d'abandonner des traitements inutiles ou risqués.

\section{Le Dr Bruno Kissling et le Prof. Fabian Krause} rapportent respectivement dans les numéros 46 et 47 du Bulletin des médecins suisses que les recherches dans la Cochrane Library ne donnent parfois aucun résultat ou alors des résultats qui ne sont plus actuels; cela n'incite pas à utiliser la Cochrane Library. Que conseillez-vous? Des mesures sont-elles prévues pour optimiser l'offre?

Avec un seul mot-clé pour effectuer une recherche, il est rare d'obtenir directement le résultat souhaité. En employant des termes standardisés comme les MeSH (Medical Subject Headings-Terms), également utilisés dans la banque de données PubMed, on aura déjà plus de succès. Par ailleurs, il est très compliqué d'actualiser les revues Cochrane existantes, car de nou- veaux résultats d'études viennent continuellement s'y ajouter. Cochrane essaie donc de fixer des priorités et de proposer de l'aide aux groupes d'auteurs, afin que les revues les plus importantes soient rédigées ou actualisées plus rapidement. Entre-temps, on peut utiliser ce que nous appelons les Living Systematic Reviews, qui intègrent en continu les nouvelles études. Ce qu'aucune autre banque de données ne propose.

\section{Quels sont, selon vous, les avantages des revues} Cochrane par rapport aux guides de pratique?

Un guide de pratique basé sur des données probantes se réfère, dans le meilleur des cas, à une ou à plusieurs revues Cochrane. C'est du moins le cas pour de nombreux guides de pratique de l'OMS. Lorsqu'un médecin qui exerce suit ce qui y est inscrit, il ne risque pas de se tromper. Mais il ne s'agit pas ici d'opter soit pour l'un, soit pour l'autre. Une série de recommandations isolées dans un guide de pratique couvre un autre besoin en informations qu'une revue systématique qui présente, de la manière la plus transparente possible, les données scientifiques prouvées dont nous disposons actuellement.

\section{Quelles différences voyez-vous entre les revues Cochrane et les rapports d'évaluation des techno- logies de santé?}

Une revue Cochrane classique sur une forme de traitement reprend de manière systématique les données probantes existant dans le monde sur les avantages et les dangers cliniques. Il en va de même pour les mesures diagnostiques ou préventives. Dans les rapports d'évaluation des technologies de santé, ces données probantes sont complétées par des analyses juridiques,

Les conclusions d'un rapport d'évaluation des technologies de santé ne peuvent être transposées à d'autres pays.

éthiques et économiques (de la santé). A cela s'ajoute une classification des faits par un organisme indépendant. Les conclusions d'un rapport d'évaluation des technologies de santé ne peuvent être transposées à d'autres pays que dans une certaine limite, alors que c'est beaucoup plus facile avec les revues Cochrane.

Les revues Cochrane reposent souvent sur un collectif de patients sélectionnés. Dans quelle mesure les résultats sont-ils pertinents pour les patients sous-représentés, par exemple les personnes atteintes de polymorbidité, les femmes, les personnes âgées? Une revue Cochrane se fonde sur les données des études qu'elle inclut. Si ces études ne reflètent qu'un 
sous-groupe de patients sélectionnés, la pertinence de la revue sera limitée. Les études se distinguent souvent par leurs critères de sélection. On parle alors d'hétérogénéité. D'un côté, cela peut être problématique pour combiner les données de plusieurs études dans une méta-analyse; de l'autre, cela permet aussi souvent de

\section{Une revue Cochrane se fonde sur les données des études qu'elle inclut.}

dire si le résultat (p. ex. la suprématie d'un traitement) peut être généralisé et s'il ne s'applique pas seulement à un groupe choisi de patients idéaux.

\section{La médecine centrée sur le patient gagne en impor-} tance. Dans quelle mesure les attentes et le point de vue des patients sont-ils pris en compte par Cochrane? Cela se passe à plusieurs niveaux: dans le choix des critères cibles (les fameux outcomes ou objectifs) permettant de mesurer l'efficacité d'un traitement, les revues Cochrane mettent la priorité sur les critères concernant les patients. En d'autres termes, la fonctionnalité, la douleur et la qualité de vie sont mises au premier plan, même si les groupes de patients des études considérées sont comparés selon un nombre important de paramètres de laboratoire.

L'autre niveau consiste à associer des patients, hommes et femmes, aux travaux de Cochrane. Pour cela, il existe le Cochrane Consumer Network, qui inclut la perspective des patients par exemple lorsqu'il s'agit de fixer des priorités thématiques pour les travaux des groupes de revues.
Selon vous, quel rôle joue la politique autour de Cochrane? Quelles pourraient être les tâches des acteurs de la santé en vue de promouvoir la Cochrane Library?

Il serait souhaitable que les arguments découlant de données scientifiques probantes trouvent un écho plus important dans les processus politiques. Aujourd'hui, sur les questions de climat, les enfants descendent dans la rue pour nous exhorter nous, adultes, de manière probante, à faire notre devoir, à savoir traduire enfin en actes les déclarations émises par les scientifiques. Il y a 20 ans, cela aurait été inimaginable.

Que les données probantes sur les questions de santé proviennent de Cochrane ou d'une autre source fiable est secondaire. L'important, c'est de les prendre suffisamment en compte quand on prend une décision. Cela ne veut pas non plus dire d'ignorer d'autres arguments tels que les préférences des personnes concer-

\section{La Cochrane Library est une source d'informa-} tion indépendante, fondée sur des données probantes, librement accessible à tous en Suisse.

nées. Nous pouvons être fiers, à juste titre, de la qualité élevée de notre système de santé, mais ce ne sera plus le cas si nous continuons à financer des mesures sans preuves suffisantes de leur efficacité et si l'ensemble du système devient inabordable. La Cochrane Library est une source d'information indépendante, fondée sur des données probantes, librement accessible à tous en Suisse. J'espère qu'à l'avenir, elle bénéficiera d'un soutien financier encore plus large.

\section{Qu'est-ce que Cochrane?}

Cochrane est un réseau mondial indépendant regroupant chercheurs, professionnels de la santé, patients et autres personnes intéressées par les questions de santé. Le réseau est alimenté par environ 11000 membres et plus de 68000 sympathisants (supporters), originaires de plus de 130 pays. A I'aide de méthodes scientifiques, ces membres préparent des informations fiables et libres de toute influence commerciale sur diverses questions ayant trait à la santé. Chaque revue Cochrane est dédiée à un thème clairement délimité. Pour traiter ces sujets, un groupe d'auteurs rassemble un corpus d'études originales répondant aux critères définis au préalable. II évalue ensuite les études retenues pour déterminer s'il existe suffisamment d'indices fiables confirmant la validité d'un traitement, d'une mesure préventive, d'un traitement ou d'un diagnostic donnés. Chaque fois que possible, les résultats individuels sont combinés en une méta-analyse. Avant d'être rendues publiques, les revues Cochrane sont évaluées dans le cadre d'un processus de révision par les pairs. Plus de 8000 revues sont aujourd'hui disponibles sur le site www.cochrane

library.com. Outre la version intégrale, très complète, divers formats condensés sont également à disposition. Format particulièrement adapté à la pratique clinique, les revues les plus pertinentes et les plus demandées sont présentées sous la forme de questions-réponses (Cochrane Clinical Answers). 